\title{
VIII.
}

Aus der medic. Klinik des Prof. A. Strü mpell in Erlangen.

\section{Ueber einen Fall \\ von Tuberculose des oberen Lendenmarkes mit besonderer Berücksichtigung der secundären Degenerationen.}

\author{
Von \\ Dr. Ludwig R. Nïller, \\ I. Assistent der medic. Klinik zn Erlangen. \\ (Mit 7 Abbildungen im Text.)
}

Das Auftreten eines grösseren Solitärtuberkels im Rlickenmark ist ein so seltenes Vorkommniss, dass es wohl gerechtfertigt erscheint, eine derartige Beobachtung ausführlicher mitzutheilen, um so mehr wenn, wie in unserem Falle, die verschiedenen Stadien der langen Leidensgeschichte durch den anatomischen Befund gut erklärt werden können, und die durch die primäre Herderkrankung hervorgerufenen secundären Strangdegenerationen mancherlei Interessantes darbieten.

Von einer Zusammenstellung und Besprechung der Literatur $\mathfrak{a b e r}$ Tuberculose des Rückenmarkes möchte ich absehen, da eine solche in erschöpfender Weise erst vor Kurzem von Schlesinger ${ }^{1}$ ) in dieser Zeitschrift veröffentlicht worden ist.

\section{Krankengeschichte.}

Die Anamnese unserer Patientin sei etwas ausfuhrlicher mitgetheilt, da sie für die Deutung der gefundenen Rückenmarksveränderungen nicht unwichtig ist.

Frau J., 40 Jahre alt, Maurerswittwe aus Schweinau. Aufgenommen in die medicinische Klinik am 6. December 1895.

1) Ueber centrale Tuberculose des Rückenmarkes. Deutsche Zeitschrift für Nervenheilkunde. Bd. VIII. 5. u. 6. Heft. Der dort gegebenen Literaturzusammenstellung ist noch hinzuzufügen die casuistische Mittheilung von $S u d e c k$, Ein Fall von Tuberkelbildung im Rückenmark. Jabrbücher der Hamburger Staatskrankenanstalten. Bd. IV. Jahrg. 1893/1894. 
Hereditäre Verhältnisse von Bedeutung werden nicht in Erfahrung gebracht. Patientin war verheirathet und hat zwei gesunde Kinder geboren. Bis vor 3 Jahren war sie ganz gesund und konnte kräftig arbeiten. 1892 traten anf einmal ohne vorhergehende oder begleitende sonstige Krankheitserscheinungen heftige Schmerzen (Brennen und Reissen) in den Beinen anf, das Gehvermögen wurde bald so erschwert, dass die Kranke $1 \frac{1 / 2}{2}$ Jahre an das Bett gefesselt war.

Nach diesem Zeitraum besserte sich aber der Zustand wieder so sehr, dass Patientin bei nur geringer Unterstützung gut marschiren konnte und schmerzfrei war.

Vor etwa 1 Jahre (also seit Ende 1894) wieder rasche Verschlim. merung, vor Allem von unten nach oben sich ausbreitende Gefühlsstörung (keine Schmerzen!!). Seit 6 Monaten ist auch das Gefühl am Bauche ganz erloschen. Blasen- und Mastdarmstörungen (Urinträufeln, Abgang des Stuhles ohne Empfindung). Dazu seit kurzer Zeit auch noch Beeinträchtigung in der willkürlichen Bewegungsfähigkeit der unteren Extremitäten. Häufig unwillkürliche Bewegungen und Zuckungen in denselben.

Mit Beginn der neuerlichen Erkrankung (vor 1 Jahre) traten heftige Schmerzen in beiden Armen auf, die für Rheumatismus gehalten wurden.

Störungen in der Motilität oder Sensibilität der oberen Extremitäten waren nicht vorhanden.

In den letzten Wochen kamen zu alledem noch psychische Erscheinungen hinzu, Wein- und Lachkrämpfe und zeitweise leichte Delirien. Am Kreuzbein entstanden grosse Decnbitalgeschwïre, die der sorgfältigsten Behandlung widerstanden.

Status praesens. Patientin ist psychisch matt, aber zur Zeit bei klarem Sensorium. An den Hirnnerven keine Störungen.

Die Untersuchung der inneren Organe ergiebt keinen von der Norm abweichenden Befund.

Die Musculatur des Thorax und der oberen Extremitäten sehr mager, ohne dentlich localisirte Atrophie. Mit den Armen und Fingern können alle Bewegungen ausgeführt werden, doch tritt bei Zielbewegungen eine deutliche Unsicherheit auf. Händedruck beiderseits recht schwach. Sehnenreflexe an den Armen deutlich vorhanden.

An der Wirbelsäule allgemeine runde Kyphose, nirgends Vorspringen von einzelnen Wirbeln; bei passiven Bewegungen des Rumpfes keine Steifigkeit, keine Schmerzen. Ueber dem Kreuzbein ein grosser Decubitus.

Bauchdecken stark gespannt, obere Bauchdeckenreflexe beiderseits lebhaft, die unteren Baucbdeckenreflexe sind nicht anszulösen.

Beine enorm abgemagert, so dass die Knochencontouren durch die Haut dentlich zu erkennen sind.

Im Hïftgelenk sind die Oberschenkel so stark adducirt, dass sie sich rechtwinklig kreazen, das rechte Bein liegt taber dem linken. Beide Obersehenkel sind in der Hüfte gebeugt; die Kniegelenke sind in spitzwinkliger Beugestellung, die Füsse stehen annähernd im rechten Winkel gegen die Unterschenkel. Es besteht vollständig motorisehe Para- 
plegie. Passiv können die Contracturen nur in geringem Grade überwunden werden.

An verschiedenen Stellen in der Haut der unteren Extremitäten grosse Decubitalgeschwïre.

Patellarsehnenreflexe beiderseits deutlich, wenn auch ohne starken Bewegungseffect. Ebenso ist an beiden Beineu der Achillessehnenreflex ganz ausgesprochen vorhanden, dagegen kein Fussclonus anszulösen.

Hautreflexe sind nicht nur durch Stechen in die Fusssohle, sondern schon durch Anfassen und Streichen der Haut zu erzielen, und zwar bewirkt Druck auf den Oberschenkel eine Dorsalflexion der grossen Zehe und des Fusses, Streichen an der Aussenseite des linken Unterschenkels eine starke, aber ziemlich träge Contraction im Biceps femoris, oft anch im Tibialis anticus. Bei entblössten Beinen sieht man häufig scheinbar spontane, ziemlich langsame Bewegungen in den Fiussen und Zehen.

Die Berührungsempfindlichkeit der Hant ist ebenso, wie die Schmerzund Temperaturempfindung im Gesicht, an den oberen Extremitäten und an der Brust gut erhalten; an der Bauchhaut findet man eine normale Sensibilität nur bis zur Nabelhöhe, von da ab werden tiefe Nadelstiche nur als Berührungen angegeben, die Schmerzempfindung ist ganz aufgehoben.

Ebenso sind die oberen Drittel der Oberschenkel ganz analgisch, für Tasteindrücke jedoch noch empfindlich. Die beiden unteren Drittel der Oberschenkel, ebenso wie die Unterschenkel und die Dorsalseite der Füsse sind vollständig anästhetisch. Patientin empfindet auch die eintretenden Reflexzuckungen nicht mehr. Dagegen reagirt Patientin anf Stiche in die Plantarseite der Füsse ansser mit unwillkürlichen trägen Contractionen in den Oberschenkelflexoren und auch mit Schmerzensäusserungen.

Während an den oberen Extremitäten und am Rumpf Kälte und Wärme tadellos unterschieden werden, hat Patientin in der unteren Bauchgegend und in den oberen Partien der Oberschenkel nur noch Kälteempfindungen, in den ubrigen Partien der unteren Extremitäten wird weder kalt noch warm empfunden.

Mit starken faradischen Strömen ist die atrophische Musculatur der Beine noch deutlich erregbar.

Urin und Stuhl geht unwillkürlich ab. Der Harn enthält weder Eiweiss noch Zucker.

Aus der weiteren Krankengeschichte sei nur mitgetheilt, dass Frau J. unter Ansteigen der Anfangs normalen Temperatur immer elender wurde. Die Decubitalgeschwüre wurden trotz der sorgfältigsten Pflege grösser, das Sensorium war meist benommen, die Kranke leidet an Verfolgungsideen und verweigert die Nahrung (wahrscheinlich Inanitionsdelirien). Die Haut der Füsse um die Malleolen wurde ödematös geschwollen.

Am 2. Januar 1896 finden wir notirt, dass ausser den Patellarreflexen auch die Hautreflexe in den abgemagerten und contracturirten Beinen noch deutlich auszulösen sind. Bei Druck auf den Fuss tritt eine langsame Anspannung der Beugemusculatur am Oberschenkel (Biceps, Semimembranosus) ein, welche auch nach Nachlassen des Druckes noch mehrere Secunden lang anhält, um dann langsam nachzulassen. Dieselben auffallend $t r a ̈ g e n$, ton ischen Hautreflexe sind durch leichte 
Nadelstiche in die Fusssohle und in die Hinterseite des Oberschenkels auszulösen, während von der Vorderfläche der Beine nur geringe oder gar keine Reflexe erzielt werden können.

Genaue Sensibilitätsprüfungen sind bei dem benommenen Zustand der Patientin nicht möglich. Der Urin wird unwillkürlich nach mehrstündigen Pausen entleert. Oft tritt unwillkürliche Harnentleerung ein, wenn die Beine der Patientin angefasst werden (erhöhte Reflexerregbarkeit der Blase).

Nach raschem Kräfteverfall und unter steten Inanitionsdelirien trat am 19. Januar 1896 der Tod ein.

Die Section ergab: Pleuritis chronica fibrinosa duplex. Tuberculosis sanata lobi superioris utriusque. Oedema pulmonum, Infiltratio glandularum bronchialium. Struma hyperplastica duplex, Atrophia adiposa hepatis, Cholelithiasis. Tuberculosis Durae matris spinalis. Tuberculosis medullae spinalis $I$ mbalis.

Das Rückenmark wurđe mir von Herrn Prof. Hauser in gütiger Weise zur mikroskopischen Untersuchung überlassen. Es sei mir gestattet, auch an dieser Stelle bierfiur meinen besten Dank auszusprechen.

Die Härtung des Rückenmarkes geschah Anfangs in Müller'scher Lösung, später in Alkohol.

Die Schnitte wurden behufs der Markscheidenfärbung nach der Weigert'schen und nach der Pal'schen Methode behandelt. Die Kernfärbung gelang am besten mit Böhmer'scher Hämatoxylinlösung. Die Axencylinder traten am dentlichsten bei der Gieson'schen Färbung hervor. Hauptsächlich da, wo die Axencylinder von den Markhüllen entblösst sind, ziehe ich die Gie s o n'sche Färbung der Nigrosin- und Goldmethode vor.

Eine kurze makroskopische Beschreibung des Rückenmarkes sei der ausführlicheren Schilderung des mikroskopischen Befundes vorangeschickt.

Die hintere dorsale (äussere) Fläche der Dura mater ist vom 4. Halswirbel bis zum 2. Brustwirbel mit den Wirbeln verwachsen. Bei der Herausnahme der Dura bleiben an der Aussenfläche derselben reichliche lockere Massen von bröckligem Granulationsgewebe haften. Der Knochen ist da, wo ihm das eben beschriebene Gewebe anliegt, vom Periost entblösst, rauh, doch gelingt es nirgends, mit der Sonde tiefer in ibn einzudringen. ${ }^{1}$ )

Die Granulationen greifen nirgends durch die Dura mater hindurch, deren In ne nflä che im Gegensatz zu der rauhen Aussenfläche am ganzen Halsmark glatt und normal erscheint. Eine Compression des Halsmarkes durch das der Dura mater aufliegende Granulationsgewebe ist makroskopisch nicht erkennbar.

Vom 2.-12. Brustnervenabgang ist die Dura mater vollständig normal, auch die Zeichnung dẹs Ruickenmarkes behält bis in das unterste Brustmark, abgesehen von der in den Hintersträngen schon makroskopisch sichtbaren aufsteigenden Degeneration, ihre vollständig normale Configuration. Allein schon in der Höhe des 12. Brustnerven sieht man auf

1) Eine Herausnahme der Wirbel war aus äusseren Gründen unthunlich. 
dem Querschnitt die ganze Gegend der Hinterstränge etwas vorgewölbt, verwaschen, von grauer Farbe, die Hinterhörner werden durch die Masse der Hinterstränge förmlich auseinander gedrängt.

$1 / 2 \mathrm{Cm}$. tiefer nimmt das Rüekenmark an Volumen allseitig t u morartig zu und bildet eine spindelförmige Verdickung, welehe erst im unteren Lendenmark, etwa $5 \mathrm{Cm}$. oberhalb des Conus terminalis der normalen Configuration wieder Platz macht. Diese Anschwellung hat in ihrer grössten Ausdehnung einen Querdurchmesser von $1 \frac{1}{2} \mathrm{Cm}$, einen Sagittaldurchmesser von $1 \mathrm{Cm}$. Auf dem Querschnitt ist die normale Zeichnung vollkommen verwischt; das Rückenmark ist in eine grauröthliche, etwas fleckig aussehende Masse verwandelt, welche in der Mitte einige gelbliche, käsige Stellen zeigt.

Entsprechend dieser Auftreibung des Lendenmarkes ist die Dura $\mathrm{mater}$ in ihren hinteren (dorsalen) Partien beträchtlich verdickt (2 $\mathrm{Mm}$.) und hier mit dem Lendenmark durch mehrfache Adhäsionen verbunden.

Zwischen der rauhen, bröckligen Aussenfläche der Dura und der Wirbelsäule findet sich reichliches, der Dura zum Theil fest anhaftendes Granulationsgewebe.

Im unteren Lendenmark und im Sacralmark ist die Zeichnung des Riickenmarkes, abgesehen von einer deutlichen absteigenden Degeneration in den PyS., wieder völlig normal.

Die mikroskopische Beschreibung der krankhaften Veränderungen im Rückenmark beginnt am besten mit der Schilderung des Gewebes in der tumorartigen Anschwellung des Lendenmarkes.

Ein Querschnitt durch das Rückenmark nach Abgang des 12. Brustnerven ist weit umfangreicher, als ein solcher durch die Halsanschwellung. Der dorsale Theil der Dura mater und die derselben an ihrer Aussenseite reichlich aufliegenden Massen sind auf den Schnitten mitgetroffen. Das Gewebe der harten Ruickenmarkshaut ist auf dem Querschnitt bis $\mathrm{zu} 2 \mathrm{Mm}$. verdickt, derb fibrös. Die Auflagerungen erweisen sich mikroskopisch als ausserordentlich zellreich, und zwar sind die kleinen protoplasmalosen, intensiv gefärbten Zellkerne zu kleinen dichten Gruppen gehäuft. Dazwischen liegen grössere Partien, die sich nicht färbten und in deren Bereich ausser trüben, scholligen Massen sich keine Zellstructur erkennen lässt (verkäste Herde). Ganz ähnlich ist das Aussehen der im Rückenmark selbst entstandenen Neubildung. Mit der Weigert'schen Methode gefärbte Schnitte aus dieser Höhe bleiben ganz blass. Nur die eintretenden und die Dura mater durchsetzenden hinteren Wurzelfasern des 1. Lumbalnerven sind noch deutlich schwarz gefärbt.

Bei der Kernfärbung (Hämatoxylin) wird der Schnitt fleckig blan. Die helleren Partien entsprechen den verkästen oder in Verkäsung begriffenen Stellen. Dieselben sind etwa von der Grösse eines Stecknadelkopfes; nur in der Mitte des Tumors liegt ein $3 \mathrm{Mm}$. langer und $1 \mathrm{Mm}$. breiter Streifen, in dem gar keine Zellstructur mehr zu erkennen ist, und der mithin einen grösseren Käseherd darstellt.

Das den makroskopisch dunkler erscheinenden Partien entsprechende Gewebe besteht aus dichtgedrängten Rundzellen. Zellkern liegt an Zellkern; in der Mitte von solchen Zellanhänfungen finden sich oft Ries enzellen, deren reichliche Zellkerne meist peripherisch gelegen sind und

Deutsche Zeitschr. f. Nervenheilkunde. X. Bd. 
deren homogenes Protoplasma sich nur wenig färbt. In der Nähe der Riesenzellen oder am Rande des in regressiver Metamorphose begriffenen Gewebes kann man oft dentlich ansgeprägte epitheloide Zellen erkennen.

Zwischen den einzelnen Zellanhäufungen ziehen zahlreiche blutstrotzende, meist quergetroffene Gefässe. Die einzelnen Knötchen selbst aber, vorzüglich das käsig zu Grunde gehende Gewebe, zeigt völligen Mangel an Capillaren.

Trotz Anwendung verschiedener Methoden ist es mir nicht gelungen, in dem oben ausführlich beschriebenen Gewebe Tuberkelbacillen nachzuweisen. Nach dem histologischen Befunde kann aber ein Zweifel über die Natur dieses Tumors nicht anfkommen: es handelt sich um tuberculöses, zum Theil in Verkäsung übergegangenes Granulationsg e w e be. Diese Neubildung hat fast den ganzen Querschnitt des oberen Lendenmarkes zerstört. Nur links aussen ist ein schmaler Rand, der von der zelligen, tuberculösen Infiltration noch nicht ergriffen ist, sondern nur aus gewuchertem Gliageweb besteht. In ihm sind durch die Giesonsche Färbungsmethode vereinzelte, lebhaft rothe Axencylinder nachzuweisen.

Es ist dies auch die einzige Stelle, in der ich mit der Pal'schen Methode schwarze Schollen und Marktrimmer finden konnte. Gut e rbalte $n$ e Markscheiden (Ringe) kann man aber auch hier nicht entdecken. Die damit von Neuem erwiesene Thatsache, dass die Axencylinder zerstörenden Einflüssen länger widerstehen, als die Markseheiden, soll später noch eingehender besprochen werden.

Wie schon in der makroskopischen Beschreibung erwähnt, reicht der Solitärtuberkel von etwas oberhalb des Abganges des 1. bis zu dem Abgang des 2. Lumbalnerven. Mikroskopisch ist in allen Höhen des Tuberkels keine wesentliche Veränderung gegenïber dem eben mitgetheilten Befunde zu constatiren.

Nach Abgang des 2. Lumbalnerven ist zwar von Granulationsgewebe nichts mehr zu erkennen, der Sehnitt ist aber trotzdem bei Markscheidenfärbung ganz blass geblieben. Nur hier und da findet man Markschollen oder grosse aufgequollene, schlecht tingirte Markscheiden. Bei Anwendung der Gieson'schen Färbung, bei der sich bekanntlich die Gliakerne blau, die Markscheiden blassgelb, die Axencylinder lebhaft roth färben, kann man in diesem, der normalen Markscheiden ganz entbehrenden Gewebe recht zahlreiche Axencylinder auffinden. Um dieselben herum sieht man freilich noch meist den Querschnitt der noch vorhandenen, aber nicht mehr färbbaren, d. h. abnorm veränderten Markscheide. Ein Unterschied zwischen grauer und weisser Substanz ist in dieser Höhe makroskopisch noch nicht zu erkennen. An der Stelle der Vorderhörner findet man vereinzelte Ganglienzellen, die aber keine Fortsätze mehr haben und abgerundet, stark pigmentirt in dem kernreichen Gewebe liegen. Das Rückenmark ist hier ausserordentlich gefässreich. Um die kleinen verdickten Gefässe liegt ein breiter, hyaliner Hof, um die grösseren Gefässe findet sich eine reichliche Rundzellenanhäufung. Im Wesentlichen besteht das Gewebe in dieser Höhe (nach Abgang des 2. Lumbalnerven) aus vermehrter Stützsubstanz. Sowohl die Gliakerne, als auch ihr feines Fasernetz sind in stärkster Wucherung begriffen. Die dem Rückenmark 
hier anliegende Pia ist dicht mit Rundzellen durchsetzt, an der Dura mater ist keine krankhafte Veränderung mehr zu erkennen.

Einen Nervenabgang weiter unten (3. Lumbalsegment) differenzirt sich die graue Substanz wieder gut von der weissen. Der Gehalt der Schnitte an normalen Markscheiden ist noch ein recht geringer, und über dem ganzen Schnitt verbreitet finden sich noch zahlreiche Marktrümmer und Myelinkugeln. In der Gegend der PyS sieht man beiderseits ein dreieckiges Feld, dessen Spitze nach innen gerichtet ist, dessen Basis bis an die Peripherie reicht. Dasselbe ist von normalen Markscheiden fast ganz entblösst. Etwas weniger vollständig, aber doch schon makroskopisch leicht erkennbar, ist der Faserausfall in den ventralen, der grauen Commissur anliegenden Partien der Hinterstränge. Nach hinten ist diese Lichtung in den Hintersträngen nicht scharf zu begrenzen.

In der Höhe des 4. Lumbalnerven (s. Fig. 1) gleicht das Rückenmark bis anf die eben erwähnten, jetzt deutlich sich abgrenzenden secundär degenerirten Partien in den Seiten- und Hintersträngen 'ganz einem normalen Querschnitt aus dieser Höhe. Die Zeichnung der granen Substanz, die Ganglienzellen, der Gehalt der weissen Substanz an markhaltigen Fasern, alles dies ist nun wieder in völlig normaler Weise vorhanden. Während der Faseraus. fall in den PyS ein fast vollständiger ist, sind die vorderen Partien der Hinterstränge zwar stark gelichtet, enthalten aber schon wieder ziemlich reichliche markhaltige Nerven. Die dorsalen Partien der Hinterstränge sind fast ganz intact. Der Querschnitt der secundären Degeneration in den Seitensträngen ist

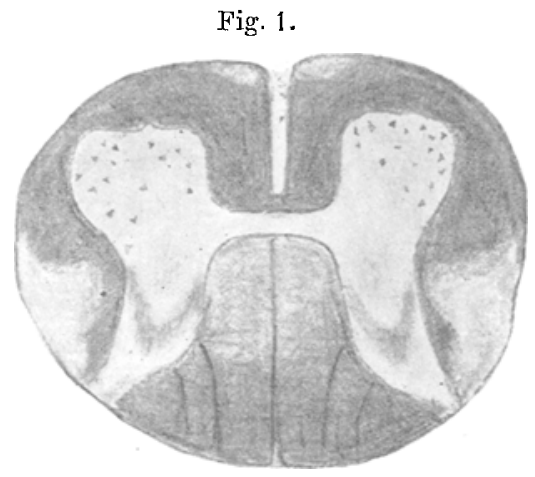

Nach Abgang des 3. Lumbalnerven. entschieden bedeutend grösser, als es bei einer absteigenden Degeneration der PyS im Anschluss an einen Gehirnherd der Fall ist. Von der Basis des degenerirten Dreieckes erstreckt sich eine deutlich degenerirte Randpartie noch weiter nach vorn und hinten (s. Fig. 1). Endlich findet sich in den Vordersträngen beiderseits nach aussen vom vorderen Ende der Fissura anterior ein kleines, deutlich abgegrenztes degenerirtes Feld (s. Fig. 1), daneben vielleicht noch eine undentliche Randdegeneration bis in die Vorder-Seitenstränge hinein.

Weiter unten, zwischen dem Abgang des 5. Lumbal- und dem des 1. Sacralnerven, ist das dreieckige Feld der degenerirten PyS schon wesentlich kleiner geworden. Doch findet sich auch hier noch die oben erwähnte Verbreiterung der Basis des im Wesentlichen dreieckigen Feldes. Die Degeneration in den Vordersträngen ist kanm noch angedentet.

In den ventralen Partien der Hinterstränge ist der höher oben so deutliche Faserausfall schon kaum mehr zu erkennen, dagegen ist jetzt neben der dorsalen Hälfte des Septum medianum pos. terius jederseits ein $1 / 3 \mathrm{Mm}$. breiter Streifen erkennbar, weleher durch den 
fast völligen Faserausfall von den übrigen vollkommen erhaltenen Partien der Hinterstränge scharf absticht. Nach vorn za sind diese schmalen degenerirten Zonen wenig scharf begrenzt, ihre laterale Grenze wird durch ein kräftig ausgebildetes Gliaseptum gebildet. Die Stützsubstanz in diesem schmalen Streifen ist deutlich verdickt, Markscheiden sind ebensowenig wie Axencylinder in demselben zu finden. Da, wo diese Degeneration des "dorso-medialen Sacralbündels" zuerst auftritt, liegt das kleine degenerirte Feld ganz hinten (dorsalwärts) zu beiden Seiten des Septum posterius, so dass es bis an die hintere Peripherie des Rückenmarkes heranreicht (Fig. 2).

Noch weiter unten in der Höhe des 3. nnd 4. Sacralnerven ist das dem Septum medianum postic. anliegende degenerirte Btindel etwas nach vorn, ventralwärts gerückt, so dass die schmalen marklosen Streifen die hintere Peripherie nicht mehr erreichen (s. Fig. 3). Die Begrenzungslinien sind nicht mehr ganz parallel dem Septum, sondern etwas nach

Fig. 2.

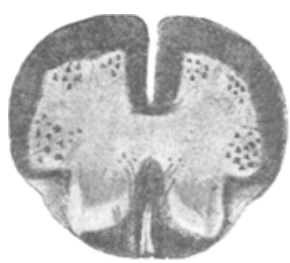

Nach Abgang des 1. Sacralnerven.
Fig. 3.

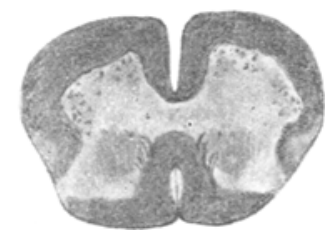

Unteres Sacralmark.

aussen convex ("ovales Hinterstrangfeld"). Die schwache Degeneration in den Seitensträngen ist hier auf zwei kleine Dreiecke lateral von den Hinterhörnern an der Peripherie des Rückenmarkes beschränkt. In den Vordersträngen ist eine deutliche Degeneration nicht mehr sichtbar. An den das Rückenmark hier zablreich umgebenden Wnrzelfasern ist ein ausgesprochener Nervenfaserausfall weder in den vorderen, noch in den hinteren Wurzeln zu erkennen.

\section{Aufsteigende Degeneration.}

Während in der Höhe des 12. Brustnervenabganges (kurz oberhalb des Tuberkels) an der dorsalen Seite der Dura sich noch reichliche tuberculöse, zum Theil verkäste Granulationen vorfinden, ist am Rückenmarksquerschnitt selbst nichts mehr von specifisch tuberculösem Gewebe vorhanden. Der Umriss der grauen Substan z ist hier wieder deutlich erkennbar, die feinen Markfasern in derselben sind ebenso wie die Ganglienzellen in den Vorderhörnern und in den Clarke'schen Säulen im Wesentlichen normal.

Anders steht es mit der weissen Substanz (s. Fig. 4). Die Hinterstränge sind in ihrer ganzen Ausdehnung degenerirt. Nur ein ganz schmaler Streifen an der medialen Seite der Hinterhörner enthält einige, den letzteren parallel verlaufende markhaltige Fasern. In den Vorderund Seitensträngen ist nur eine innere, der grauen Substanz anliegende Zone fast ganz normal erhalten. Die äusseren Partien bilden eine breite, 
Ueber einen Fall von Tuberculose des oberen Lendenmarkes u. s. w. 281

stark degenerirte Randzone (s. Fig. 4), welche sich fast um die ganze Peripherie der Vorder- und Seitenstränge erstreckt. Die Gegend der PyS enthält beiderseits noch verhältnissmässig mehr normale Fasern, als die nach vorn sich daran anschliessenden Partien der Seitenstränge. Die hinteren Abschnitte der Vorderstränge sind fast normal. Die Lissauerschen Fasern sind auf der einen Seite ziemlich gut erhalten, auf der anderen sehr stark gelichtet.

In den Hintersträngen und in den Randpartien der Seiten- und Vorderstränge, kurz überall da, wo Nervengewebe im Untergang begriffen ist, finden sich zahlreiche meist quergetroffene Gefässe, umgeben von zahlreichen runden Zellkernen, welche sich in nichts von den zerstreut liegenden Zellkernen unterscheiden.

Die vorderen $W$ urzelfasern sind in dieser Höhe tadellos erhalten, die hinteren nur zum Theil, einige sind recht arm an Markfasern.

Auf Schnitten etwas höher oben (Höhe des 11., 10. und 9. Brustnervenabganges) erhalten die dorsalen Partien der Seitenstränge

Fig. 4.

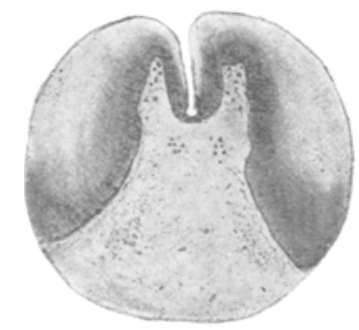

Brustmark kurz vor Abgang des 12. Brustnerven.
Fig. 5.

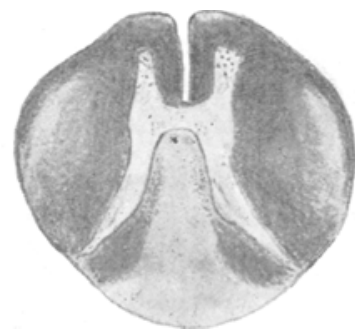

Nach Abgang des 9. Brustnerven.

(Gegend der PyS und KIS) ihren normalen Markgehalt. Dagegen befindet sich beiderseits vor den PyS ein nicht ganz seharf abgrenzbares Gebiet, dessen Markfasern bedeutend reducirt sind. Von den Vorderhörnern ist es durch ein ziemlich breites Bündel normal markhaltigen Gewebes getrennt (s. Fig. 5). Nach vorn und hinten läuft es etwas spitz zu. Die vordere Spitze wird durch eine schmale normale Randzone von der äusseren Peripherie des Rückenmarkes getrennt, während weiter hinten (aber noch vor den PyS) die Degenerationszone den Rückenmarksrand erreicht. Die Vorderstränge haben sich wieder fast ganz erholt. Nur an ihrem vorderen inneren Winkel za beiden Seiten des vorderen Endes der Fissura anterior ist ein ganz geringer Faserausfall bemerkbar. Die Degeneration in den Hintersträngen wirrd, je höher hinauf, durch die eintretenden Wurzelfasern immer mehr eingeengt.

Auf den Schnitten durch das Brustmark sieht man diffus vertheilt, besonders reichlich aber in den Seitensträngen, leere, rundliche Lücken (erweiterte Lymphspalten?). Die perivasculären Lymphräume um die kleineren und mittelgrossen Gefässe sind stark erweitert.

Die Lichtung in den mittleren Partien der Seitenstränge ist auch im oberen Brustmark (s. Fig. 6) so dentlich, dass sie auf Prä- 
paraten mit Markscheidenfürbung mit blossem A uge gut erkannt werden kann. Sie hat etwa die Form eines Dreieckes, dessen Spitze gegen die Vorderhörner gerichtet ist, dessen Basis der seitlichen Peripherie des Rückenmarkes anliegt. Am $R$ ande des Rückenmarkes wird aber die Zahl der gut erbaltenen markhaltigen Fasern wieder entschieden dichter. Die Degeneration in den Goll'schen Strängen reicht im ganzen Brustmark nach vorn bis an die hintere Commissur und zeigt an ihrem vorderen Ende die bekannte flaschenförmige Verbreiterung (s. Fig. 6). In den Vordersträngen scheint auch hier eine geringe Degeneration am vorderen Rande zu bestehen.

Im Gegensatz zu der vollständigen Degeneration des Nervengewebes in den Goll'sehen Strängen mit secundärer Gliawucherung handelt es sich in den beschriebenen Partien der Seitenstränge nur um einen Faserausfall, eine starke Lichtung; die Gliakerne sind hier nicht wesentlich vermehrt, das Gliagerüst aber doch etwas verdickt.

Der anf Schnitten durch das untere $\mathrm{Halsmark}$ mitgetroffenen Dura mater liegen wieder reichliche tuberculöse Granulationen

Fig. 6.

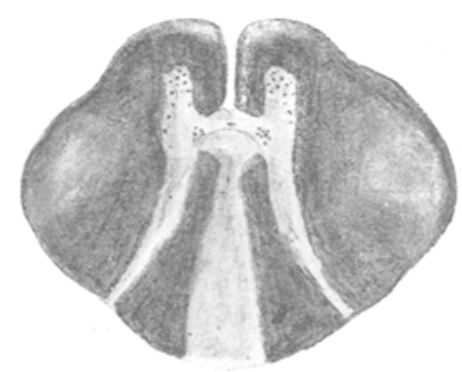

Brustmark nach dem Abgang des 2. Dorsalnerven.
Fig. 7 .

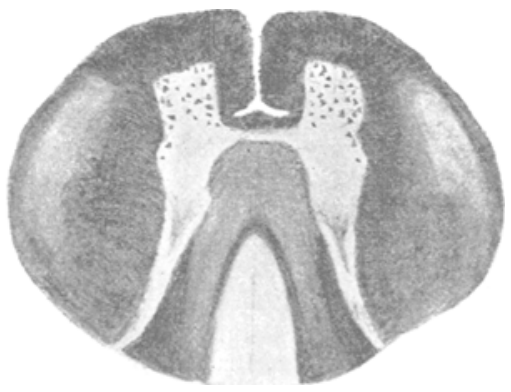

Malsmark kurz nach Abgang des 3. Cervicalnerven.

auf, die mikroskopisch mit Sicherheit als solche zu diagnosticiren sind. Die Innenfläche der Dura mater ist auch hier vollständig glatt. Die zwischen der verdickten Dura und der Medulla spin. gelegenen hinteren Wurzeln sind arm an intacten Nervenfasern, enthalten dafür reichliche Marktrïmmer und Markschollen. Auf dem Rückenmarksquerschnitt sind keine tuberculös entzündlichen Veränderungen zu constatiren, doch ist die Farbe der ebenso wie die vorhergehenden Schnitte mit Weigert'schem Hämatoxylin behandelten Präparate weniger schwarz, fast grau zu nennen; augenscheinlich haben die Markscheiden unter dem geringen Druck durch die Granulationen doch etwas gelitten.

Die oben beschriebene Degeneration in den mittleren Partien der Seitenstränge ist auch im Halsmark recht deutlich.

Neu aufgetreten ist in den Hintersträngen neben dem völligen Faserausfall in den Goll'schen Strängen, die hier nicht mehr bis an die hintere, graue Commissur reichten, eine schwächere Degeneration in den Burdach'schen Strängen. Vollkommen normal sind in diesen nur die „hinteren äusseren Felder". Die Wurzeleintrittszonen und noch mehr die 
vorderen Partien zeigen einen starken Faserausfall. An den medialen vorderen Ecken der Vorderstränge ist das kleine degenerirte Feld deutlich sichtbar. Die grane Substanz ist normal.

Im mittleren und oberen Halsmark (s. Fig. 7) in einer Höhe, in der an der Aussenfläche der Dura keine Granulationen mehr haften, ist die Lichtung in den Seitenstranggrundbündeln auf einen schmalen, nach innen von dem Gow ers'schen Anterolateraltract gelegenen Streifen redu. cirt (s. Fig. 7). Doch erstreckt sich ein geringer, schwer abgrenzbarer Faserausfall anch weiter nach hinten in die Gegend der KIS hinein.

In den Hintersträngen ist der schmale Keil der Goll'schen Stränge völlig entblösst von normalem Markgewebe, reicht aber nur mehr bis etwas über die Hälfte des Sept. med. postic. nach vorn. Die Degeneration in den Burdach'schen Strängen ist noch dentlich; sie wird aber nach a ssen durch die neu hinzutretenden normalen Wurzelfasern eingeengt. In den vordersten Abschnitten der Hinterstränge ist die Degeneration nur schwach angedeutet. Hintere änssere Felder normal. In den Vordersträngen ist hier eine deutliche Degeneration nicht mehr sichtbar.

Die Medulla oblo $\mathrm{ngata}$ ist nicht mikroskopisch untersucht worden.

Eine mikroskopische Untersuchung verschiedener peripherischer Nerven (Peronei u. a.) ergab keinen von der Norm abweichenden Befund. In den Museulis peroneis hat sich zwischen die einzelnen Muskelfibrillen viel Fettgewebe eingedrängt. Die Querstreifung ist an den meisten Fibrillen recht gut, an anderen daneben liegenden aber gar nicht mehr zu erkennen. Neben zahlreichen normal breiten Fasern finden sich deutlich verschmälerte. Die Zahl der Muskelkerne ist entschieden vermehrt.

Wie hat man nun die einzelnen soeben beschriebenen Veränderungen des Rückenmarkes zu erklären, und wie kann man sie untereinander und mit den zu Lebzeiten der Kranken beobachteten klinischen Erscheinungen in Einklang bringen?

Der Beginn der Erkrankung mit Schmerzen in den Beinen und starker Schwäche in denselben weist darauf hin, dass die ersten in Betracht kommenden anatomischen Veränderungen in der Bildung der tuberculösen Granulationen an der A ussenfläcbe der Dura mater lumbalis bestanden.

Diese tuberculösen Granulationen comprimirten die hinteren Wurzeln des Lumbalmarkes und verursachten dadurch die heftigen Schmerzen, welche hauptsächlich in die unteren Extremitäten localisirt wurden. Die Parese der Beine ist auf eine Beeinträchtigung des Rückenmarkes selbst durch Compression und Lymphstau ung zurückzuführen.

Nach Verlauf von 18 Monaten schrumpft das Granulationsgewebe zum grössten Theil zu einer bindegewebigen Schwiele (die Dicke der der Dura beträgt an der Dorsalseite des Lendenmarkes bis zu $11 / 2 \mathrm{Mm}$.). 
Mit der hierbei eintretenden Verminderung der Compression werden auch die motorischen Bahnen wieder gut wegbar, so dass die Kranke wieder gehen kann und fast ganz schmerzfrei ist. Allein, nach etwa Jahresfrist tritt eine neue rasche Verschlimmerung ein: 0 hne Schmerz en entwickelt sich bald wieder eine von unten nach oben bis zur Nabelhöhe sich ausbreitende Gefühlsstörung, dann völlige Paraplegie der Beine, schliesslich Blasen- und Mastdarmlähmung. Der taberculöse Process hat von der Dura mater aus das Rïckenmarksgewebe selbst befallen und dort zuerst die dorsalen Partien, die Hinterstränge, ergriffen (Gefühlsstörungen). Dass die Zerstörung dieser Bahnen keine Schmerzen verursacht, ist nicht auffallend. Wissen wir doch, dass alle intraspinal gelegenen Geschwülste oder Destructionsprocesse (Syringomyelie u. a.) keine erheblichen sensiblen Reizerscheinungen verursachen. Erst später wurden durch die ventralwärts vorschreitende tubereulöse Entzlindung auch die Seiten- und Vorderstränge geschädigt und grösstentheils zerstört. Zu der Sensibilitätsstörung gesellten sich die von Neuem auftretenden Lähmungserscheinungen in den Beinen, in der Blase und im Mastdarm hinzu. Die häufigen unwillkürlichen Bewegungen der unteren Extremitäten sind entweder auf eine directe entzündliche Reizung motorischer Fasern oder vielleicht auch auf eine reflectorisehe Erregung derselben zuritickzuführen.

Die Thatsache, dass auch nach dem Eintritt der motorischen und sensiblen Paraplegie die Patellarreflexe, wenn auch schwach, so doch noch deutlich auszulösen waren, spricht dafür, dass dieser Reflex unterhalb des 1. Lumbalsegmentes zu Stande kommt. Aber auch die Hautreflexe in den unteren Extremitäten waren nicht erloschen. Auffallend war nur die eigenthümliche Langsamkeit und Trägheit der reflectorisch hervorgerufenen Muskelcontractionen. Nimmt man für diese Reflexe nicht einen Reflexbogen unterhalb des erkrankten Querschnittes im oberen Lendenmark an, sondern, wofür ja bekanntlich manche Erfahrungen sprechen, einen Reflexbogen weiter oben im Gehirn, so müssten wir voraussetzen, dass in der Höhe des Tuberkels keine vollständige Unterbrechung aller Fasern stattfand. In der That konnten auch in der tuberculösen Neubildung selbst mit Hülfe der Gie s o n'schen Färbung einzelne übrig gebliebene Axencylinder nachgewiesen werden, während die Markscheiden in der ganzen Ausdeh nung des tuberculös erkrankten Querschnittes völlig zerstört sehienen. Wir finden somit von Neuem den Satz bestätigt, dass die Markscheiden gegenüber schädlichen Einwirkungen weit weniger widerstandsfähig sind, als die Axencylinder. Auch der Umstand, dass die Sensibilität in den Beinen, wenn auch stark herabgesetzt, so doch 
keineswegs erloschen war, spricht mit Sicherheit dafür, dass sensible Reize noch irgendwie den Weg durch die tuberculöse Neubildung hindurch gefunden haben. Wie so häufig bei Querschnittserkrankungen des Ruickenmarkes, war aber die motorische Leitung im Gegensatz zur sensiblen Leitung vollständig unterbrochen, so dass also eine völlige Paraplegie der Beine bestand.

Die beftigen Schmerzen in den Armen endlich, welche sich mit Beginn der neuerlichen Erkrankung einstellten, und die von der Patientin als "Rheumatismus" angesprochen wurden, sind wohl sicher auf die Wurzelcompression durch die Duratuberculose in der Gegend des unteren Halsmarkes zurückzuführen. Die psychischen Erscheinungen der letzten Wochen, die Weinkrämpfe und Wahnideen müssen wir als Inanitionsdelirien auffassen.

Von den anatomischen Befunden in unserem Falle verdient zunächst die Tuber culos e der Rü ckenmarksubstanz selbst noch einmal eine besondere Erwähnung. Wie selten ein derartiger Befund von "centraler Tuberculose" im Rückenmark ist, wurde schon oben hervorgehoben. Es fragt sich nun, auf welchem Wege die Infection des Rückenmarkes mit Tuberkelbacillen in unserem Falle erfolgt ist. Wie soeben erörtert, glauben wir, dass die Tuberculose an der Aussenseite der Dura mater (welche ihrerseits vielleicht mit einem tuberculösen Herd in der Wirbelsäule zusammenhing, was abęr nicht bestimmt nachgewiesen werden konnte) der Entwicklung des Riuckenmarktuberkels vorberging. Eine directe Ausbreitung der Tuberculose per contiguitatem von der Dura mater auf das Riickenmark hat aber bestimmt nicht stattgefunden, denn die Innenseite der Dura war hier wie überall glatt, die Dura selbst nirgends mit dem Rïckenmark verwachsen. Als Infectionsweg muss man daher aller Wahrseheinlichkeit nach die zwischen der harten Riickenmarkshaut und der Medulla spinalis bestehenden Ly $\mathrm{mphcommunicationen} \mathrm{ansehen.} \mathrm{Hierfür}$ spricht namentlich auch, dass die Tuberculose des Rückenmarkes an der hinteren Peripherie derselben angefangen hat, hier finden wir die stärksten regressiven Erscheinungen (ein grösserer Käseherd). Gerade an der hinteren Peripherie des Duralsackes sassen aber auch tuberculöse Granulationen. Von hinten her ist also wahrseheinlich die Invasion der Tuberkelbacillen ins Rückenmark hinein erfolgt. Dann hat sich die specifische tuberculöse Neubildung in solchem Grade weiter entwickelt, dass in der Böhe der Austrittsstellen des 1. und 2. Lumbalnerven scheinbar der ganze Rückenmarksquerschnitt durch typisches, zum Theil verkästes tuberculöses Granulationsgewebe ersetzt war. 
Ober- wie unterhalb des Tuberkels ist das Nervengewebe noch auf eine mehr oder weniger lange Strecke zerstört, die normale Rückenmarksstructur ist hier nicht mehr durch tuberculöse Granulationen, sondern durch gewuchertes Gliagewebe ersetzt. Dies ist die Zone der sogenannten ,traumatischen Degeneration" oder der umgebenden Erweichung, wie wir sie in der Peripherie der meisten Geschwülste antreffen. Diese Zone reicht caudalwärts entschieden weiter, als nach oben. Kurz oberhalb des Tuberkels (Abgang des 12. Brustnerven) sind die vorderen und seitlichen Partien der grauen Substanz schon mit einem schmalen Rande gut erhaltener Markfasern umgrenzt, die Zeichnung der grauen Substanz selbst tritt scharf hervor, die Ganglienzellen in derselben sind deutlich erkennbar. Dagegen lassen sich ein Segment tiefer, als das untere Ende des Tuberkels (Austritt des 3. Lumbalnerven) im Rückenmarksquerschnitt noch keine normalen Markscheiden erkennen, ebensowenig hat sich die graue Substanz erholt. An der Stelle der Vorderhörner sind in dem dichten, kernreichen Gliagewebe nur vereinzelte grössere, stark körnig pigmentirte Zellen sichtbar, die augenscheinlich als Ganglienzellen anzusehen sind.

Von besonderem Interesse in unserem Fall scheint mir das oben näher geschilderte Verhalten der secundären Degenerationen zu sein. Wir hatten hier Gelegenheit, bei einer fast den ges a m mten Querschnitt des obersten Lumbalsegmentes mit Einschluss der grauen Substanz zerstörenden Erkrankung die für unsere Erkenntniss der Zusammensetzung der Rückenmarksstränge so wichtige Untersuchung dieser secundären Veränderungen vorzunehmen.

Beginnen wir mit der Betrachtung der secunndären absteigenden Degenerationen, so finden wir dieselben in drei verschiedenen Gebieten (vgl. oben Fig. 1,2 und 3).

1. Degeneration der Pyramiden-Seitenstrangbahnen und gewisser Fasern, die sich unmittelbar nach vorn und hinten an den äusseren peripherischen Rand der PyS-Zone anlagern.

2. Degeneration eines kleinen umschriebenen Faserbündels in den Vordersträngen (beiderseits in der Nähe des vorderen medialen Winkels derselben). Ganz geringe absteigende Randdegeneration in den Vorder-Seitensträngen.

3. a) Eine kurze absteigende Degeneration im ventralen Hinterstrangfeld̃.

b) Weiter abwärts deutliche absteigende Degeneration des sogenannten dorso-medialen Sacralbündels.

Ueber die Degeneration der PyS ist nichts Besonderes hin- 
zuzufügen, sie entspricht den allgemein bekannten Verbältnissen. Nur ist bemerkenswerth, dass der degenerirte Querschnitt im Lendenmark entschieden weit grösser ist, als der gewöhnliche Querschnitt einer bei einem Gehirnherde abwärts degenerirten PyS. Dieses wechselnde Verhalten der absteigenden Degeneration in den Seitensträngen, je nachdem der primäre Herd im Gehirn oder im Rückenmark selbst liegt, ist bekanntlich schon von Bouchard beschrieben worden. Es scheint, als ob sich zu den von den motorischen Centralwindungen herstammenden langen PyS-Fasern andere Fasern hinzugesellen, die weiter unten im Rückenmark selbst entspringen und vielleicht die Bedeutung sogenannter Commissurenfasern haben.

Das kleine, bis zur Höhe des 5. Lumbalnerven abwärts degenerirte Feld in den medialen Winkeln der Vorderstränge erseheint auf den ersten Blick als letztes Ende der Pyramiden-Vorderstrangbahn. Doch handelt es sich wahrscheinlich um andersartige Fasern (s. u.). Eine absteigende, weiter reichende $R$ anddegeneration ${ }^{1}$ ) ist nur in geringem Grade angedeutet.

In den Hintersträngen findet sich zunächst unterhalb des Herdes eine sehr deutliche Degeneration im ventralen Hinterstrangfeld (Fig. 1). Sie reicht aber nicht weit nach abwärts, ist unmittelbar unterhalb des Herdes (3. Lumbalnerv) am stärksten, um bereits zwischen dem Abgange des 5. Lumbal- und 1. Sacralnerven ganz aufzuhören. Die Bedeutung dieser abwärts degenerirenden Fasern in den Hintersträngen ist nicht bekannt. Wahrscheinlich gehören sie nicht zu den unmittelbaren Fortsetzungen der hinteren Wurzelfasern, da bekanntlich gerade dieses ventrale Hinterstrangfeld im Lendenmark bei der Tabes, selbst in den fortgeschrittensten Fällen nicht degenerirt (Strủ pell). Auf seine absteigende Degeneration bei Querschnittsunterbrechung des oberen Lendenmarkes hat man meines Wissens bisher nicht geachtet. Vielleicht entspricht dieselbe der von Schultze gefundenen kurzen absteigenden Degeneration in den "kommaförmigen Feldern" des Hals - und oberen Brustmarkes.

Sehr deutlich war in unserem Fall die in letzter Zeit wiederholt beschriebene secundäre Degeneration des "dorso medialen Sacralbündels", eines kleinen Fasergebietes dessen secundäre Degeneration wiederum in auffallendem Gegensatz zu der Thatsache steht, das dasselbe bei dem primären Degenerationsprocess der hinteren Wurzelneurone bei der Tabes dorsalis in der Regel völlig verschont

1) Vergl. Marie, Vorlesungen über die Krankheiten des Rückenmarkes Deutsche Ausgabe. S. 37 . 
bleibt (Strümpell, Flechsig, Pineles u. a.)، Die Angaben über die specielle Lage des Feldes wechseln ein wenig. Wenn es sich hierbei, wie ich glaube, nur um individuelle Abweichungen und nicht um verschiedene Gruppen desselben Fasergebietes handelt, so muss man die Annahme machen, dass es sich um ein langes, in den Hintersträngen abwärts verlaufendes Fasersystem handelt. Denn wie zuerst Dax enberger ${ }^{1}$ ) bei einem Fall aus der hiesigen Klinik beschrieben hat, findet man die absteigende Degeneration dieses medialen Hinterstrangfeldes (dessen beide Theile aus den beiden Hintersträngen zusammen das „ovale Feld“ Flechsig's bilden) auch schon bei einer Compression im unteren Halsmark. Besonders eingehend hat sich $\mathrm{Hoche}^{2}$ ) neuerdings mit der Frage der absteigenden Degeneration in den Hintersträngen beschäftigt. Auch er fand das ovale mediane Feld im Lendenmark degenerirt bei einer Querschnitts-Unterbrechung in der Höhe des 7. Dorsalnerven und einer solchen im unteren Halsmark und schliesst daher, ähnlich wie Gombault und Philippe ${ }^{3}$ ), dass es sich nicht um Faserabkömmlinge aus den Spinalganglien, sondern ans viel böher gelegenen Strangzellen in den Hinterhörnern handelt.

Ueber die Gruppierung der Fasern in den verschiedenen Höhen des Rückenmarkes herrschen noch manche Widerspriiche. In unserem Fall war die Degeneration dieses Bündels kurz unterhalb der Querschnittszerstörung nicht nachzuweisen; bis zum 4. Lumbalsegment reicht die Degeneration der kurzen Bahnen im ventralen Hinterstrangsfeld. Von da ab erst erkennt man eine schmale Lichtung beiderseits neben der hinteren, der Peripherie zuliegenden Hälfte des Septum medianum posticum (siehe Fig. 1). Dieser Saum rỉckt weiter unten (im Sacralmark) mehr nach vorn, der grauen Commissur näher, die Bewegungslinien werden bogenförmig („ovales Feld") (siehe Fig. 2.)

In neuester Zeit ist von $\mathrm{Bruce}$ und $\mathrm{Muir}{ }^{4}$ ) eine Beobachtung mitgetheilt worden, welche mit unserem Falle viele Berührungspunkte darbietet. Es handelte sich um eine Fractur und Luxation des 12. Brustwirbels, wodurch der Uebergang vom Brust- bis zum Lendenmark zerquetscht worden war. Die Autoren fanden, dass die in dem oberen Lumbarmark unregelmässig über die Hinterstränge vertheilten Fasern sich im unteren Lenden- und im Sacralmark zu einem

1) Deutsche Zeitschr. für Nervenheilkunde. Bd. IV. S. 146, Fig. 4 und S. 148.

2) Archiv für Psychiatrie. Bd. XXVIII. S. $521 \mathrm{ff}$, und Neurolog. Centalblatt. 1896. Nr. 4. S. 154. Daselbst auch die übrige Literatur.

3) Archives de médecine expérimentale. 1894. Tome VI.

4) Brain 1896. 
Bündel vereinigen, welches sich nach vorwärts in einem schmalen Band bis zur hinteren Commissur erstreckt. Im unteren Sacralmark wird die degenerirte Zone, ganz ebenso wie dies $O$ berste in er beschreibt, gegen die Peripherie etwas umfangreicher und sitzt dort breitbasig auf, während in unserem Fall der schmale markfasernlose Streifen neben dem Sept. post. im Conus terminalis nach vorn rückt.

Zweifellos handelt es sich aber um dasselbe Strangsystem, dessen Lagerung nur eben bei verschiedenen Individuen eine etwas verschiedenartige ist.

Ueber die Functionen der Fasern, welche das dorso-mediale Sacralbündel " oder, wie es der Länge seines Faserverlaufes wegen von Edinger wohl passender bezeichnet worden ist, den "Tractus cervico-lu mbalis dorsalis" bilden, lassen sich natürlich einstweilen noch kaum Vermuthungen aufstellen. Immerhin liegt der Gedanke nahe, dass Fasern, welche bis ins unterste Sacralmark herabreichen, irgendwelche Beziehungen zu den daselbst liegenden Centren für die Blasen-, Mastdarm- und Geschlechtsfunctionen haben. Weiter gehende Vermuthungen wären aber einstweilen noch völlig zweifelhaft. -

Wir gehen jetzt zur Betrachtung der secundären aufsteigenden Degenerationen über. Oberhalb des Solitärtuberkels erholt sich die graue $\mathrm{Substanz}$ am raschesten wieder. Sie ist bald in ihren vorderen und seitlichen Partien wieder umgeben von einer schmalen Zone normaler markhaltiger Fasern. Dann aber finden wir weiterhin die folgenden aufsteigenden Degenerationen:

1. auf eine kurze Strecke eine Randdegeneration in den Vorder- und Seitensträngen.

2. eine Degeneration der Goll'schen Stränge.

3. eine lange aufsteigende Degeneration in den Grund bündeln der Seitenstränge.

4. eine schwache aufsteigende Degeneration an den medialen Ecken der Vorderstränge, die sich aufwärts bis in das mittlere Halsmark hinein verfolgen lässt.

Ehe wir auf diese Degenerationen im Einzelnen noch etwas näher eingehen, ist zuvor noch besonders hervorzuheben, dass die KlS-Bahnen und das Gowers'sche Bündel in unserem Fall keine aufsteigende Degeneration zeigen. In Bezug auf die KIS-Bahn hat diese Thatsache nichts Auffallendes, da deren Ursprungszellen bekanntlich in den Clarke'schen Säulen des unteren Brustmarkes liegen, welche von der tuberculösen Neubildung verschont geblieben 
waren. Bemerkenswerth ist aber der Umstand, dass auch der Gower's sche Tractus antero-lateralis keine Degeneration zeigte, obwohl der Ursprung dieser Fasern weiter tiefer im Lendenmark (nach Obersteiner in den Vorderhornzellen desselben) liegen soll. Hier sind also noch weitere Untersuchungen nothwendig.

Die aufsteigende Randdegeneration in den Vorder- und Seitensträngen reicht nur bis zur Höhe des 10. Dorsalsegmentes hinauf.

Die Degeneration der Goll'schen Stränge bietet nichts Bemerkenswerthes dar. Sie entspricht unseren jetzigen Anschauungen iiber die Zusammensetzung dieser Partien der Hinterstränge aus hinteren Wurzelfasern. Hier mag auch gleich noch erwähnt werden, dass im Hals mark eine neue schwache Degenerationszone (s. Fig. 6) nach aussen und vorn von den fast völlig entarteten Goll'schen Strängen hinzutritt.

5. Diese neue Degeneration hängt wohl sicher nicht von der Zerstörung des oberen Lendenmarkes ab, sondern ist die Folge einer Compression der hinteren Cervicalwurzeln durch die daselbst an der äusseren Oberfläche der Dura entstandenen tuberculösen Granulationen (s. o.)

Die aufsteigende Degeneration des kleinen Feldes an der medialen vorderen Ecke der Vordersträng e entspricht anscheinend demselben Felde, welches auch abwärts einen deutlichen Faserausfall zeigt. Dieser Umstand und auch die Lage des Feldes haben uns verhindert, die abwärts degenerirten Fasern als Py-Vorderstrangbahn anzusprechen. In der Regel liegt letztere auch weiter dorsalwärts neben dem medialen Rande der Vorderstränge. Aehnliche, nur*noch etwas weiter ausgedehnte secundäre auf- und absteigende Degenerationen in den Vordersträngen hat auch Hoche (a. a. O.) in seinen beiden Fällen gefunden.

Von besonderem Interesse erscheint uns aber in unserem Fall die secundäre aufsteigende Degeneration in den Seitensträngen, in einer so deutlich umschriebenen Form, wie sie bisher noch nicht näher beschrieben ist. Ein Blick auf die Figuren $3-6$ zeigt, dass es sich um eine ziemlich scharf abgegrenzte Zone handelt, welche continuirlich bis ins Halsmark hinein verfolgt werden kann und sicher im Wesentlichen weder dem Gebiet der PyS, noch der KIS oder dem Gowers'schen Bündel, sondern den sog. Seitenstrangresten angehört, die nach vorn von den PyS und nach innen von den KIS gelegen sind.

Wie bekannt, weisen alle neuen anatomischen Untersuchungen, vor Allem diejenigen von Edinger, darauf hin, dass hier centripetale, 
sensible Fasern liegen, welche aus den Strangzellen der (gekreuzten) Hinterbörner stammen und das zweite sensible Neuron darstellen. Ist diese Anschauung richtig, so musste auf die Zerstörung jener Zellen in den Hinterhörnern stets eine secundäre Degeneration in den gekreuzten Seitenstrangresten folgen. Dass diese Voraussetzung bisher noch nicht oft thatsächlich erwiesen ist, mag wohl damit zusammenhängen, dass die graue Substanz, insbesondere der Hinterhörner, sich der gewöhnlichen Querschnittsläsionen und vor Allem den Compressionen gegenüber verhältnissmässig sehr widerstandsfähig erweist.

Um. so interessanter war die in einer früheren Beobachtung aus unserer Klinik von $\mathrm{K}$ orb beschriebene Thatsache, dass bei der gliösen Degeneration eines Hinterhornes in dem Seitenstrang der anderen Seite eine deutliche secundäre Degeneration nachweisbar war ${ }^{1}$, eine Degeneration, welche mit der oben erwähnten Anschauung über den Verlauf der sensiblen Bahnen im Rückenmark gut iubereinstimmte. Auch L. Mann hat neuerdings (D. Zeitschr. für Nervenheilk. Bd. X. S. 62) anatomische Befunde veröffentlicht, welche für die Annahme einer sensiblen Leitung in den Seitensträngen sprechen.

In unserem Fall handelt es sich um eine Querschnittsunterbrechung, bei welcher die tuberculöse Neubildung ebenfalls die graue Substanz und ganz sicher auch diejenige der Hinterhörner im höchsten Grade zertört hatte. Daher erscheint uns auch hier die Annahme durchaus wahrscheinlich, dass jene Zerstörung der Strangzellen in den Hinterhörnern des oberen Lumbalmarkes nothwendiger Weise eine aufsteigende Degeneration in jenen Theilen der Seitenstränge zur Folge haben musste, welche wir auch thatsächlich entartet fanden. Insofern ist unsere Beobachtung, wie die Korb'sche, eine Bestätigung jener oben erwähnten neueren Anschauungen über den weiteren Verlauf der sensiblen Bahnen in den Seitensträngen des Rückenmarkes.

Zum Schlusse möchte ich für die gätige Unterstützung und vielfache Anregung bei der Abfassung dieser Arbeit meinem hochverebrten Lehrer und Chef, Herrn Professor v. Strümpell, herzlichen Dank sagen.

1) Deutsche Zeitschr. f. Nervenheilkunde. Bd. VIII. S. 369. Fig. 4. 